\title{
Intervalos de cuasi-descomponibilidad y propiedades emergentes ${ }^{*}$
}

\author{
(Intervals of Quasi-Decompositionality and Emergent Properties) \\ Emilio Cáceres VÁzquez y Cristian Saborido
}

Received: 30/01/2016

Final Version: 06/04/2016

BIBLID 0495-4548(2017)32:1p.89-108

DOI: $10.1387 /$ theoria. 15718

RESUMEN: La noción de emergencia acompaña a la Filosofía de la Ciencia desde finales del siglo xix, afirmando que en algunos sistemas existen propiedades a ciertos niveles que no pueden deducirse de las propiedades de los componentes de estos sistemas tal y como son observadas en niveles más fundamentales. A lo largo del siglo xx las caracterizaciones de este concepto de emergencia se han apoyado en cuatro pilares: impredecibilidad, novedad, restricción y causación descendente. Estos pilares están ligados a la asunción de una jerarquización de la realidad en niveles de organización. En este trabajo intentamos demostrar que puede explicarse la naturaleza de las propiedades (aparentemente) emergentes utilizando la noción de cuasi-descomponibilidad propuesta originariamente por Herbert Simon.

Palabras clave: Emergencia, reduccionismo, mecanicismo, epistemología.

ABSTRACT: The notion of emergence has accompanied philosophy of science since the late XIX century, claiming that in some systems there are properties in certain levels that cannot be deduced from properties of their components as seen in more fundamental levels. Throughout the $\mathrm{xx}$ century, emergence has been characterized by four pillars: unpredictability, novelty, restriction and downward causation. These four pillars have been related to the assumption of a hierarchical order of reality in different levels of organization. In this paper, we show that it is possible to explain the nature of the (apparent) emergent properties through the nearly-decomposability criterion introduced by Herbert Simon.

Keywords: Emergence, reductionism, mechanisms, epistemology.

\section{Introducción}

La idea de que toda la materia está formada exclusivamente por sus componentes materiales está asentada en nuestro pensamiento científico. No obstante, existe un rechazo a pensar que el comportamiento de esa materia, especialmente la considerada viva, está determinada por sus elementos. Este planteamiento, resumido en el aforismo el todo es más que la suma de las partes, ha supuesto la división del estudio de la materia en dos enfoques contrapuestos, el de aquellos que piensan que los todos pueden reducirse a sus partes y el de aquellos

* Queremos agradecer su desinteresada colaboración a Álvaro Martínez del Pozo, Catedrático de Bioquímica y Biología Molecular de la Universidad Complutense de Madrid, y a Jorge Alegre-Cebollada, jefe de grupo del Single-Molecule Mechanobiochemistry lab del CNIC por su colaboración a la hora conocer los aspectos biológicamente más actuales. 
que afirman que tienen «algo más» que hace que emerjan nuevas características no derivables desde sus componentes.

La existencia o la inexistencia de esta sinergia es un problema en busca de solución desde hace más de 160 años cuando John Stuart Mill hablara por vez primera, al menos de forma explícita, de leyes heteropáticas (Mill 1843, 269) Estas leyes estaban en principio vinculadas a la química, pero pronto pasaron a formar parte de la biología sobre todo a raíz de la publicación de la obra de Charles Darwin y su compromiso con el gradualismo. El fin del vitalismo, el desarrollo de la biología como una ciencia analítica o el fallo del reduccionismo (Bunge 2004, 191) han supuesto hitos importantes para comprender las diferentes caracterizaciones que se han dado de la idea de la emergencia y que han llevado este concepto hasta nuestros días. A lo largo de todo el siglo $\mathrm{xx}$, la emergencia ha avanzado ligada a la existencia de niveles de organización y, aunque no se puede considerar un concepto con una definición única, si se han identificado cuatro pilares en los que todas las caracterizaciones de lo emergente se sustentan, a saber: a) la impredecibilidad, o imposibilidad de deducción de las propiedades de los todos a partir de sus partes, b) la novedad cualitativa, o la existencia genuinas nuevas propiedades en niveles superiores, c) la restricción o realizabilidad múltiple, o la limitación impuesta por los niveles macro sobre los diferentes niveles micro y d) la causalidad descendente, o la influencia causal del todo en sus partes (Klee 1984).

Actualmente, hay dos tendencias principales a la hora de tratar la emergencia. Por un lado está el enfoque, cercano a la noción más clásica, que aboga por la existencia de un cierre causal (casual closure) que hace especial énfasis en la auto-organización y en la influencia del todo sobre las partes (Maturana y Varela 1973). Por otro, cercano al reduccionismo, la visión mecanicista mantiene algunas ideas surgidas de la emergencia como la existencia de niveles cualitativamente diferentes, la realizabilidad múltiple y una cierta causación descendente (Machamer, Darden y Craver 2000).

En este trabajo se va a argumentar en contra de las propiedades emergentes y de la existencia objetiva de los niveles de organización a la vez que se describe un reduccionismo heurísticamente modulado con fines eminentemente prácticos. Para ello, partiendo del concepto de criterio de cuasi-descomponibilidad de Herbert Simon mostramos que es posible redefinir las ideas de novedad, causación descendente y restricción sin necesidad de apelar a propiedades emergentes.

\section{Los pilares fundamentales de la emergencia}

El término 'emergencia' ha auspiciado diferentes posturas filosóficas ya desde que George Lewes lo acuñara en 1874 (Lewes 1874, (II), 412), extrapolando la idea desde una explicación de las propiedades de los compuestos químicos por parte de Mill a la filosofía postdarwinista (Morgan 1923). El término se ha tratado en tantos ámbitos y con tantas connotaciones que no existe un criterio unificado que lo defina. Muchas han sido las aproximaciones, destacando las de Mario Bunge (Bunge 2004), Clauss Emmeche (Emmeche, Köppe, y Stjernfelt 1997), William Wimsatt (Wimsatt 2000), Timothy O’Connor (O'Connor 1994), Richard Campbell y Mark Bickhard (R. J. Campbell y Bickhard 2011), Michael Silberstein y John McGeever (Silberstein y McGeever 1999) o David Blitz (Blitz 1992). Son también varios los análisis metateóricos que se han propuesto para dar cuenta de las características de las diferentes concepciones y para tratar de ofrecer una clasificación de los tipos de emergen- 
cia más utilizados, como el trabajo ya clásico de Robert Klee (Klee 1984) o el más reciente de Tero Piiroinen (Piiroinen 2014). Estos trabajos subrayan que la clave de la idea de la emergencia estriba en su origen histórico y en su evolución temporal.

Así, muestran cómo la formulación por parte de Jakob Schleiden y Theodor Schwann de la teoría celular en 1839 supuso un primer paso en la visión reduccionista de la vida. Fisiólogos de la época llegaron incluso a declarar que «en el mundo orgánico no hay otras fuerzas en actividad que las fisicoquimicas conocida» (Mayr 2006, 94). Y esto en relación no solo con los aspectos fisiológicos sino también con los «del pensamiento y del lenguaje, del libre albedrio, etc.» (Garrison 1922, 162). Aun así, la ciencia estaba impregnada de vitalismo (Mayr 2006, 95) y consideraba que los fenómenos biológicos eran manifestaciones directas del alma y por lo tanto no explicables materialmente (Emmeche, Köppe y Stjernfelt 1997, 89). Esta postura vitalista llegó hasta principios del siglo XX, con Hans Driesch como último vitalista influyente $(1997,91)$, hasta que J.S Haldane declaró en 1931 que «los biólogos han abandonado casi unánimemente el vitalismo como creencia aceptable» (Mayr 2005, 30). Sin embargo, este abandono no supuso la aceptación del mecanicismo sino la introducción de un nuevo programa, el organicismo, que si bien aceptaba la idea básica de que los componentes y sus propiedades fisicoquímicas eran los responsables de todas las funciones vitales, su influencia se disipaba conforme se ascendía en complejidad, pues la totalidad influía causalmente en las partes. E.W. Ritter, introductor del término «organicismo», lo expresaba así en 1919: «los todos están tan relacionados con sus partes que no solo la existencia del todo depende de la cooperación ordenada y la independencia de sus partes, sino que el todo ejerce además un cierto grado de control determinista de sus partes» $(2005,31)$.

Esta idea de que el todo es más que la suma de las partes, y no simplemente la suma de estas, procede de la posición de Mill que distinguía entre causas que se combinan dando un resultado aparentemente novedoso y aquellas genuinamente nuevas. Las leyes homopáticas, para lo predecible, y las heteropáticas, para lo impredecible, se complementan dando lugar a resultados generalmente homogéneos y particularmente heterogéneos, de forma que se establece una especie de brecha antes y después de la cual, todo puede predecirse (Mill 1843, 269). Aun así, Mill aclara que «no somos, al menos en nuestro estado actual de conocimiento, capaces de prever qué resultado seguirá a partir de una nueva combinación hasta que hayamos intentado el experimento especifico» $(1843,267)$ dejando abierta una interpretación del concepto de ley heteropática más cercano a una irreductibilidad contingentemente epistemológica (Piiroinen 2014, 143) que a una emergencia al uso. Esta idea de Mill es llevada por Lewes al campo de lo vivo proponiendo la subordinación de la Física y la Química a la Biología y tratando al organismo como un todo (Lewes 1877, 21-22). Lewes amplía la consideración de emergencia a lo psíquico y basa en la emergencia la concepción de un mundo discontinuo, con cambios cualitativos, contrario a la visión darwinista gradual y cuantitativa (Blitz 1992, 80-81). Así, más allá del cuño del término, plantea varios de los principios que recogerán los denominados emergentistas británicos: la novedad cualitativa, la imposibilidad de reducción, las propiedades de los todos frente a sus partes y la jerarquía de las ciencias y de sus respectivos niveles de estudio. Fue Edward Spaulding uno de los primeros en buscar una razón a la emergencia al afirmar que «el análisis involucra la relación todo-parte» (Spaulding 1912, 158), dando una importancia decisiva a la organización, especialmente en los todos orgánicos (1912, 237-247). Además, alude a la influencia de los niveles superiores sobre los inferiores, configurando un esbozo de lo que posteriormente se denominará «causación descendente $>(1918,450-451)$. 
A principios del siglo xx la idea de emergencia ya está perfilada de forma que podrá ser usada para tratar de responder a ciertos problemas de la filosofía de la biología, algo que según los críticos del emergentismo no se logró hacer (Baylis 1929). Por ejemplo, Lloyd Morgan utiliza el nuevo concepto como eje de una teoría de la evolución con la que intentaba resolver el gradualismo darwiniano. Desde un enfoque filosófico, este problema era grave (Blitz $1992,1)$, pues si la evolución trabaja gradualmente no puede haber ninguna separación entre dos características cualesquiera que no pueda rellenarse por un número finito de términos. Aquí es donde Morgan sitúa la emergencia haciendo énfasis en la novedad como algo más que un mero reordenamiento de los componentes desde un enfoque naturalista y rechazando explícitamente la entelequia de Driesch y el élan vital de Bergson (Morgan 1923, 1-2).

Paralelamente, Samuel Alexander propone una emergencia de niveles con cualidades genuinas pero conservando propiedades resultantes. Cada nivel tenía su propio y peculiar proceso de comportamiento en virtud de su organización y su complejidad (Alexander 1920, 45-46), algo que puede entenderse como leyes de nivel, que no son deducibles ni sirven para predecir el comportamiento de los niveles previos ni posteriores. Alexander declaraba no conocer la razón de la emergencia $(1920,63)$, y aunque rechazaba lo sobrenatural introdujo un aspecto teleológico al afirmar que la organización está dirigida hacia la perfección que supone la deidad $(1920,353)$, que por influir en los niveles previos puede leerse como un antecedente de la causación descendente. También aboga por la necesidad del análisis top-down, pues aunque no pone en duda la posibilidad de determinación y de predicción, afirma que no podrá hacerse de forma completa. Así, un calculador laplaciano sería capaz de determinar la micro-estructura de todo lo existente, pero esto sería incompleto pues no habría incluido las propiedades emergentes que no pueden conocerse a menos que se experimenten $(1920,328)$.

En la misma década de 1920, A. C. Broad hace una primera formalización de la teoría de la emergencia con el fin de dilucidar entre la existencia de uno o varios tipos de sustancia material (Broad 1925, 22), es decir, entre las posiciones monistas y pluralistas $(1925,38)$. En este contexto, la emergencia es un punto intermedio que elimina los problemas del mecanicismo biológico y del vitalismo sustancial como formas de explicación monista y dualista clásicas respectivamente (Blitz 1992, 117). Este vitalimo emergente parte de la existencia de ciertos todos formados por distintos componentes $\mathrm{A}, \mathrm{B}$ y $\mathrm{C}$ en una relación $\mathrm{R}$, tales que todos aquellos que cumplen la misma relación $\mathrm{R}(\mathrm{A}, \mathrm{B}, \mathrm{C})$ tienen las mismas propiedades características. Añade que los mismos componentes pueden formar otras relaciones diferentes, por ejemplo $\mathrm{S}(\mathrm{A}, \mathrm{B}, \mathrm{C})$ de forma que tendrán otras propiedades diferentes. Las propiedades de cada todo no pueden ser deducidas del completo conocimiento de las propiedades aisladas de A, B y C ni de otros todos diferentes a R (A, B, C) (Broad 1925, 61). Broad pone ejemplos, pero no proporciona explicaciones.

Hecha esta introducción, vamos a seguir el análisis que Robert Klee (Klee 1984) hizo de la emergencia con el fin de establecer cuales son los pilares fundamentales que supuestamente la sustentan. Su idea consiste en contrastar la noción de emergencia con la idea de que en una estructura organizada en niveles de complejidad creciente, los niveles superiores son deducibles de los inferiores, a lo cual denomina micro-determinismo. Klee parte de un artículo de Stephen C. Pepper (Pepper 1926) quién intentó dilucidar si las propiedades emergentes eran algo especial o meros epifenómenos. Pepper hablaba de la emergencia como de algo caracterizado por la existencia de unas marcas (marks) en los niveles superiores de organización que los distinguen de los niveles inferiores por el hecho de ser más com- 
plejos. Klee se propone buscar estas marcas y para ello se pregunta si lo que emergen son leyes, como piensan Meehl y Sellars (Meehl y Sellars 1956), Kekes (Kekes 1966) o Campbell (D.T. Campbell 1974), o son restricciones que condicionen los niveles, como piensan Weiss (Weiss 1970) y Pattee (Pattee 1971). Continúa analizando la aparición impredecible e irreductible de novedades genuinas, entendidas como hacía Kekes, es decir, como regularidades ontológicas que no están presentes en los niveles inferiores (Kekes 1966, 360). Esta concepción, que Klee llama emergencia nómica, se basa en que la materia viva se comporta de forma diferente a la inerte en virtud de su organización (Klee 1984, 46).

Tras esto, Klee pone su foco en la consideración de que desde el nivel inferior surgen ciertas constricciones (constraints) $(1984,45)$ que condicionan el nivel superior. Esta idea de límite procede de las condiciones de contorno que Michael Polanyi propuso como limitación de los posibles desarrollos del nivel macro a partir del nivel micro (Polanyi 1968). Para Polanyi es la información contenida en el ADN la que establece las condiciones dentro de las cuales puede desarrollarse la vida. Pero esa información, a pesar de estar en la secuencia, es independiente de su composición física, o en sus propias palabras, «debe ser tan físicamente indeterminado como la secuencia de las palabras lo está en una página impresa. Como la disposición de una página impresa es ajena a la química de la página impresa» (Polanyi $1968,1309)$ Esta idea del auto-cierre de lo vivo como característica diferenciadora frente a lo inerte, ha sido el germen de otros conceptos posteriores como la autopoiesis (Maturana y Varela 1973), el cierre semántico (Pattee 1982), el cierre de causalidad eficiente (Rosen 1985) de Rosen, o la obra de Kauffman (Kauffman 1993), y goza de amplio reconocimiento en la biología teórica actual (Etxeberría y Umerez 2006). Polanyi lo ejemplifica con el desarrollo embrionario argumentando que el ADN establece unas restricciones ambientales, dentro de la madre, que limita los grados de libertad y condiciona la correcta formación del embrión ${ }^{1}$.

Klee desglosa todos los aspectos anteriores llegando a cuatro características que considera distintivas de la emergencia de una propiedad $\mathrm{P}$ a partir de una micro-estructura MS. Bastaría una de ellas para que se pueda hablar de emergencia.

1. P, en principio, es impredecible a partir de MS (i.e. desde un completo conocimiento teórico de MS en el límite de la indagación científica); o

2. P es nueva con respecto a MS; o

3. MS exhibe un mayor grado de variación y fluctuaciones que aquellos del nivel de organización donde P ocurre, la constante y perdurable presencia de $\mathrm{P}$ en el sistema no parecería completamente determinada por MS; o

4. P tiene una directa y determinante influencia sobre, al menos, alguna de las propiedades en MS. (Klee 1984, 48)

1 Resulta evidente que la relación entre la visión auto-organizativa en biología y la cuestión del emergentismo en filosofía de la ciencia necesita de un tratamiento independiente. Limitándonos a los propósitos de este trabajo, queremos señalar que la caracterización teórica de la auto-organización está íntimamente ligada a la de la causación internivel, la cual es tratada directamente en nuestro artículo. Según nuestra interpretación (véase sección 4d), el auto-mantenimiento es un fenómeno apreciable exclusivamente desde un enfoque que atiende a un nivel mesoscópico. De aquí que la auto-organización pueda verse como una propiedad de nivel y, por consiguiente, dependiente en último término de las propiedades de sus elementos. 
Concretando el análisis de Klee, podríamos denominarlas impredecibilidad, novedad, restricción o realizabilidad múltiple y causación descendente.

Klee intenta explicar estos pilares, pero afirma que no hay un único enfoque que pueda explicarlos completa y convincentemente $(1984,51)$. Lograrlo es el objetivo de los siguientes apartados de este trabajo.

\section{El criterio de cuasi-descomponibilidad de Herbert Simon}

En 1962 Herbert Simon en su artículo clásico sobre la complejidad distinguía, en su disquisición acerca de las relaciones entre los subsistemas de una estructura jerarquizada, entre aquellos a los que denominaba sistemas descomponibles, en los que las relaciones entre sus subsistemas eran claramente distinguibles, y los que llamaba sistemas cuasi-descomponibles (nearly-decomposable systems), en los que dichas relaciones eran no tan claramente discernibles si bien no despreciables. Desde una perspectiva teórica, los sistemas cuasi-descomponibles se caracterizan en que en el corto plazo, el comportamiento de sus subsistemas componentes es aproximadamente independiente entre sí, mientras que en el largo plazo, dependen solo de forma agregativa (Simon 1962, 474). En su descripción de este tipo de sistema, propone un ejemplo en el que partimos de una casa con un aislamiento térmico perfecto, formada por habitaciones bien aisladas subdivididas en cubículos mal aislados, cada uno de los cuales cuenta con un termómetro. Al principio del experimento, los cubículos y las habitaciones tienen temperaturas diferentes, pero pasado un tiempo la temperatura comenzará a homogeneizarse. Primero lo hará entre cubículos debido a su mal aislamiento, posteriormente se igualará la temperatura entre habitaciones, pues a pesar del aislamiento, lo están de forma deficiente. Así, el sistema casa será cuasi-descomponible en subsistemas habitación y estos a su vez en subsistemas cubiculo (1962, 474). Los valores de flujo térmico se podrían representar en una matriz de datos como descripción del sistema cuasi-descomponible. El flujo entre cubículos será mayor que el flujo entre habitaciones, por lo que según donde pongamos nuestro límite, los cubículos serían un ejemplo de cuasi-descomponibilidad, mientras que las habitaciones serían descomponibles. Simon habla de un valor $\varepsilon$ que decidimos arbitrariamente y que nos sirve como delimitador. De hacerlo así, veríamos que en un corto plazo, la diferencia de temperatura entre cubículos habría desaparecido, por lo que disponer de un termómetro en cada uno de ellos resultaría superfluo y valdría con uno por habitación $(1962,475)$. Resulta obvio que si, en vez de utilizar un $\varepsilon$ o criterio de cuasi-descomponibilidad utilizamos otro, podríamos decir que los subsistemas habitaciones serían cuasi-descomponibles. Si en vez de utilizar un solo criterio, usamos dos, $\varepsilon_{1}$ y $\varepsilon_{2}$, tendremos la distinción entre dos subsistemas cuasi-descomponibles anidados.

Supongamos que ampliamos el campo de observación y miramos desde una perspectiva más amplia, de forma que la vivienda de Simon, su sistema aislado, forme parte de un grupo de cuatro viviendas adosadas, que a su vez forman parte de una urbanización de cuatro de esos adosados. Ahora podemos determinar diferentes criterios de cuasi-descomponibilidad $\left(\varepsilon_{n}\right)$ según el subsistema al que nos queramos referir y tendríamos varios $\varepsilon_{\mathrm{n}}$ que distinguirían cubículos, habitaciones, casas, adosados, etc. Así tendríamos que cada subsistema cuasi-descomponible estaría delimitado por dos $\varepsilon_{n}$ y sería definible por lo que podríamos llamar intervalos de cuasi-descomponibilidad $\mathrm{I}_{(i, j)}=\left[\varepsilon_{i}, \varepsilon_{j}\right]$. Con esta nomenclatura, 
tendremos cubículos o $\mathrm{I}_{(0,1)}=\left[\varepsilon_{0}, \varepsilon_{1}\right]$, habitaciones o $\mathrm{I}_{(1,2)}=\left[\varepsilon_{1}, \varepsilon_{2}\right]$, casas o $\mathrm{I}_{(2,3)}=\left[\varepsilon_{2}, \varepsilon_{3}\right]$, adosados o $\mathrm{I}_{(3,4)}=\left[\varepsilon_{3}, \varepsilon_{4}\right]$ y la urbanización $\mathrm{I}_{(4,5)}=\left[\varepsilon_{4}, \varepsilon_{5}\right]$. Todos serían sistemas cuasi-descomponibles y por tanto se influyen de forma no despreciable.

Simon parte de una situación de equilibrio que evoluciona sin injerencias, por ello, llega un momento en el que es suficiente con un termómetro en cada habitación y pasado un tiempo, uno para la casa, adosado o urbanización. Esto es así porque se alcanzaría un equilibrio estático al formar parte de un sistema aislado. Pero si en cada cubículo hay un radiador se establecerá un equilibrio dinámico de manera que habrá un gradiente térmico centrado en los radiadores. Es decir, habrá una diferencia continua y la ubicación de solo un termómetro en cada cubículo, habitación, vivienda, adosado o urbanización dependerá de una decisión arbitraria, determinada por algún tipo de interés, bien explicativo, bien predictivo, bien metodológico, etc. Si la finalidad es conocer cómo influye la temperatura de cada casa en la urbanización, observaremos solo en el intervalo $I_{(2,3)}=\left[\varepsilon_{2}, \varepsilon_{3}\right]$, promediando su temperatura sin importar sus gradientes internos ni las temperaturas de sus habitaciones y cubículos, a pesar de que la temperatura está determinada, en última instancia, por ellos. Como efecto no deseable de esta simplificación, aparecería una artificial uniformización del sistema, pudiéndose pensar que los distintos intervalos tienen idéntico comportamiento, efecto más marcado cuando más complejo sea el sistema.

Simon considera la casa como un sistema aislado, es decir, perfectamente descomponible, cuando estrictamente no existen tales sistemas. Es como si pensara en un valor límite de $\varepsilon$ por encima del cual los sistemas pasaran de cuasi-descomponibles a perfectamente descomponibles. Sin embargo, no da ninguna razón que lo justifique. Si ampliamos más el caso y pensamos en un barrio formado por urbanizaciones y un pueblo formado por barrios, es cierto que la influencia de cada cubículo en muy pequeña, pero no es despreciable, pues aunque es una cantidad mínima comparada con la procedente del sol, es la responsable de que la temperatura en los pueblos sea sensiblemente mayor que en una zona deshabitada. Si imaginamos un pueblo como éste en un imaginario lugar sin sol, resulta evidente que la diferencia entre el pueblo y las afueras serían importantes. Por lo tanto, la segunda condición de Simon para los sistemas cuasi-descomponibles, «a largo plazo, el comportamiento de cualquiera de los componentes depende unicamente de modo agregativo del comportamiento del resto de los componentes» (Simon 1962, 474), no se satisface mientras los sistemas sigan generando desigualdad.

En la revisión que hace Simon de los sistemas físico-químicos $(1962,475)$ se plantea la aplicación de un criterio basado en la diferencia energética entre enlaces covalentes y débiles. De esta manera, en función del valor de $\varepsilon$ que elijamos, podemos discriminar las asociaciones. Si situamos $\varepsilon$, como dice Simon, justo por debajo del valor energético del enlace covalente, estableceremos que las moléculas son sistemas cuasidescomponibles, mientras que aquellas uniones basadas en enlaces más débiles, son interacciones intermoleculares. Por esto consideremos el $\mathrm{H}_{2} \mathrm{O}$ como una molécula, mientras que la interacción entre varios hidrógenos y oxígenos por enlaces covalentes y de hidrógeno $\left(\mathrm{H}_{2} \mathrm{O}\right)_{n}$, lo vemos como un conjunto de moléculas y no como una macromolécula. Esta distinción si bien es relativamente clara en los enlaces covalentes, no lo es tanto en los de tipo iónico y ni en los metálicos. Simon determina el criterio de cuasi-descomponibilidad en base a la energía de enlace, pero puede escogerse cualquier criterio en función del fin perseguido. 
Así, en función de $\varepsilon_{n}$ habrá un «nivel fundamental aproblemático» (Machamer, Darden y Craver 2000, 13) o microscópico $F$, un nivel macroscópico $M$ y múltiples niveles mesoscópicos anidados $m_{i}, m_{i}, \ldots m_{n}$. De esta manera la relación entre los subsistemas superiores y los inferiores es simplemente composicional y por lo tanto, la relación entre dos niveles diferentes es de identidad. Esto sugiere que, de igual manera que la casa de Simon era el conjunto de los cubículos agrupados en habitaciones, un ser humano es un conjunto de átomos agrupados en moléculas, en células, en tejidos, en órganos y en aparatos o sistemas. Pero no solo eso, sino que mirando en el otro sentido, es un componente de poblaciones, ecosistemas y biomas, siendo un punto intermedio de un conjunto de sistemas cuasi-descomponibles anidados ${ }^{2}$.

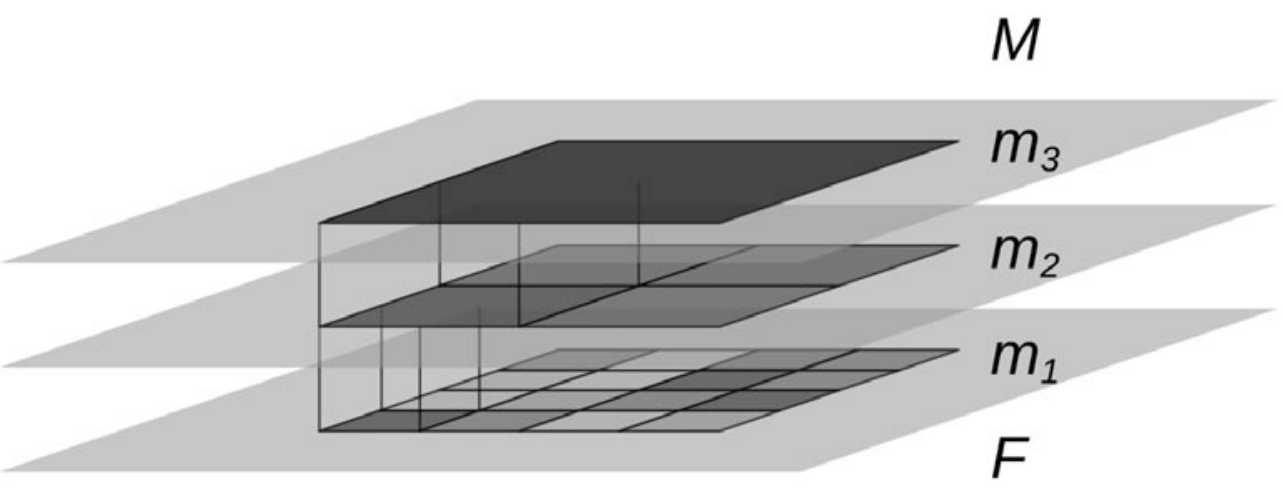

Ilustración 1. Niveles de organización

Los intervalos de cuasi-descomponibilidad no solo delimitan horizontalmente los niveles de organización de la materia, sino que determinan verticalmente las diferentes entidades existentes en cada nivel, incluso elementos que debido a su naturaleza química no aumentan de complejidad. Si definimos un $\varepsilon_{i}$ en base al valor de energía del enlace covalente, definiremos el nivel molecular, pero también nos separará las moléculas como entes individuales. De igual manera, si ampliamos el $\varepsilon_{i}$ distinguiremos el nivel celular y las células individuales, el

2 Cuando hablamos de un nivel aproblemático o fundamental $F$ nos referimos, como puede suponerse, al nivel de las propiedades físicas; nivel que, independientemente de las complicaciones derivadas de los nuevos desarrollos de la Física es considerado habitualmente como el nivel último que constituye la base para un reduccionismo ontológico. Esto va en la línea de lo defendido explícitamente por autores como Machamer, Darden y Craver (Machamer, Darden, y Craver 2000, 13) y Glennan (Glennan 1996, 50). Desde el punto de vista de la Física, Roger Penrose aclara la diferencia entre el nivel cuántico y el clásico (Penrose 1991, 374-375), señalando explícitamente que las dificultades de la Física actual no suponen realmente un reto para el enfoque reduccionista. Tal y como dijo Paul Dirac, uno de los fundadores de la mecánica cuántica: «The underlying laws necessary for the mathematical theory of a large part of physics and the whole of chemistry are thus completely known, and the difficulty is only that exact applications of these laws lead to equations which are too complicated to be soluble» (Dirac 1929, 714) tomado de (Mitchell 2012, 172). Por otro lado, López Corredoira también hace un interesante análisis de esta cuestión aquí: (López Corredoira 2004) 
nivel de individuo y los individuos individuales, el nivel de población y las poblaciones individuales, etc. Sin embargo, conforme ascendemos hacemos discriminaciones arbitrarias en función de un análisis previo top-down que condiciona la observación. Así, si estamos hablando de poblaciones humanas, eliminamos los organismos no-humanos y los no-organismos, como el aire o el agua; si pensamos en organismos humanos, eliminamos las células no humanas y las no-células, y así sucesivamente, denominando entorno a todo lo que eliminamos, lo que supone una simplificación enorme.

Un aspecto importante a destacar es el relativo al estudio de los niveles. Como ya se ha señalado, la selección del nivel depende del valor del $\varepsilon_{\mathrm{i}}$, y dado que éste es arbitrario, su elección debe obedecer a algún criterio definible. Sin que esto signifique que no pueda existir otro principio, proponemos el uso de un criterio heurístico, y por tanto dependiente del para qué de la observación. Si el objeto del estudio es, por ejemplo, el plegamiento de una proteína, podemos pensar en un $\varepsilon_{i}$ que nos permita discriminar entre macromoléculas, mientras que si el fin de una investigación es analizar la relación de apareamiento dentro de una población, la elección heurística de $\varepsilon$ nos tiene que facilitar discernir entre individuos. Sin embargo esta elección no es sencilla, pues para comprender el plegamiento de una proteína debemos tener en cuenta elementos de niveles inferiores, como pueden ser protones determinantes del $\mathrm{pH}$, de la misma forma que para entender el comportamiento de apareamiento hay que entender las bases fisiológicas de la reproducción.

Sea cual sea el criterio de elección de $\varepsilon$, su función como límite superior del intervalo de cuasi-descomponibilidad va a establecer ciertas entidades discriminables como individualidades con sus propias características que deberán ser descritas de manera adecuada, para lo que debe existir una disciplina encargada de tal tarea. Así, siguiendo con los ejemplos anteriores, habrá una ciencia que abarque el estudio del apareamiento y por lo tanto del nivel de población, la etología y otra dedicada al estudio del plegamiento de las proteínas y en general del estudio de las moléculas biológicas, la biología molecular. Cada una de estas ciencias trabajará dentro del campo de estudio determinado por la elección del intervalo de cuasi-descomponibilidad y tomará como su todo a esta partición. Sin embargo, en su estudio considerará las partes componentes que estime oportunas para poder explicar el funcionamiento de este todo. Así, la etología, además de las poblaciones considerará a los individuos y su fisiología mientras que la biología molecular ampliará su foco hasta las pequeñas moléculas y los átomos, como ya propusiera Mario Bunge, aunque con una intención diferente (Bunge 1973, 41-43). De aquí podemos extraer la idea de que cada ciencia, además de su propio $\varepsilon$, usará como criterios de sub-cuasi-descomponibilidad $\left(\varepsilon_{i}^{\text {sub }}\right)$ aquellos de las ciencias situadas en niveles inferiores. Igualmente, las ciencias encargadas del estudio de niveles mesoscópicos deberán considerar los niveles inmediatamente superiores para comprender la influencia de sus todos en los otros todos, y de manera análoga tomaran los $\varepsilon_{i}$ de las ciencias de los niveles superiores como criterios de supra-cuasi-descomponibilidad $\left(\varepsilon_{i}^{\text {supra }}\right)$. De esta manera, con la elección de los niveles en los cuales una ciencia tiene competencia secundaria se establece lo que puede denominarse como intervalo ampliado de cuasi-descomponibilidad $\mathrm{IA}_{(i, j)}=\left[\varepsilon_{i}^{\text {sub }}, \varepsilon_{j}^{\text {supra }}\right]$. 


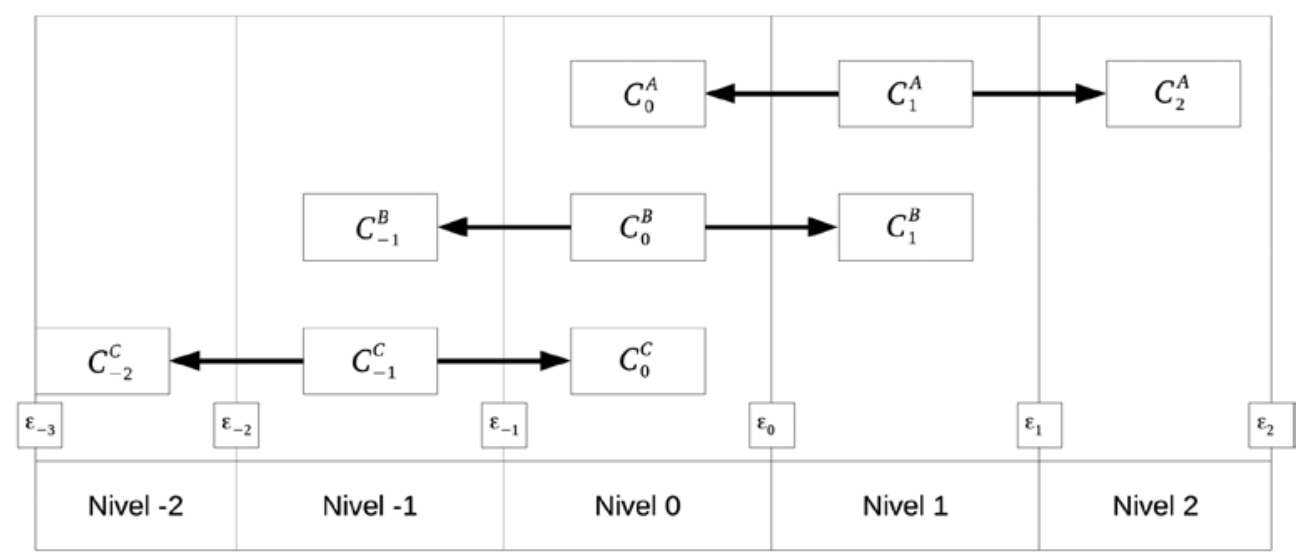

Ilustración 2. Niveles y ciencias especiales

En la ilustración 2 se puede ver cómo quedaría estructurada la materia en función de la elección de varios $\varepsilon_{i}$. Si elegimos valores entre $\varepsilon_{-2}$ y $\varepsilon_{2}$ estaremos haciendo una partición de la materia en niveles entre dos valores de $\varepsilon$. Así, el intervalo $\mathrm{I}_{(-2,-1)}=\left[\varepsilon_{-2}, \varepsilon_{-1}\right]$ nos define el nivel -1 , el $\mathrm{I}_{(-1,0)}=\left[\varepsilon_{-1}, \varepsilon_{0}\right]$ el nivel 0 y por último, el intervalo $\mathrm{I}_{(0,1)}=\left[\varepsilon_{0}, \varepsilon_{1}\right]$ establece el nivel 1 . De cada nivel se encarga por consiguiente una ciencia, en este caso, las ciencias $C, B$ y A respectivamente. Cada ciencia indagará por debajo y por encima de su nivel con el fin de conocer mejor su objeto de estudio y necesitará determinar por una parte, cuáles son sus subniveles y por otra, de qué supranivel es componente. Por esto, la ciencia A, además de usar su criterio $\varepsilon_{1}$ tendrá en consideración los niveles inmediatamente inferior y superior, y por lo tanto contará con un intervalo ampliado de estudio $\mathrm{IA}_{(-1,2)}=\left[\varepsilon_{-1}{ }^{\text {sub }}, \varepsilon_{2}{ }^{\text {supra }}\right]$.

Una vez definido el intervalo de competencia para cada disciplina, así como su ampliación, cada ciencia debe realizar una descripción de su objeto de estudio acorde a lo observable desde ese nivel, determinando así un sistema. Cada ciencia estudiará su propio intervalo, lo que consiste en distinguirlo de su entorno y describirlo estructural y funcionalmente. Esta descripción debe hacerse en función de la metodología de la ciencia en cuestión y utilizando una terminología propia, lo que implica el uso de propiedades detectables desde su nivel mediante sus métodos de investigación. Por lo tanto, tratará su intervalo como si de una caja negra se tratara, tomando solo aquellos inputs y outputs que considere necesarios para la finalidad del estudio, estableciendo un sistema modelo, haciendo una simplificación heurística de la realidad. Durante la descripción del sistema será posible detectar regularidades que podrán o no ser catalogadas como leyes.

Esta modelización, es decir, la descripción de un intervalo de cuasi-descomponibilidad en función de las propiedades de ese intervalo según la metodología de la ciencia de ese nivel, implica una simplificación importante. Un nivel determinado, por ejemplo el nivel 1, puede visualizarse como una caja que debe abrir la ciencia encargada de su estudio, por ejemplo la ciencia A. Al hacerlo, verá que su interior está ocupado por otras cajas cerradas formadas por sistemas del nivel inferior 0 , de cuyo estudio se encarga la ciencia B. A su vez, estas cajas estarán llenas de nuevas cajas cerradas que se corresponden al nivel -1, responsa- 
bilidad de la ciencia C. Este anidamiento de cajas terminaría en los citados nivel F, por debajo, y nivel M, por encima. Cuando la ciencia A va a describir fenómenos de su nivel, no entra a analizar cada una de las cajas-subsistema, sino que se conforma con ciertos datos entrada-salida medidos desde su nivel y por lo tanto con valores promediados, o proporcionados por la ciencia $\mathrm{B}$, que habrá hecho lo mismo en lugar de analizar la caja del nivel C. Por lo tanto, la mera toma de datos implica una pérdida de información asumida que influirá de forma importante en la observación de regularidades y en el establecimiento de leyes. Además, no todas las variables que forman parte de dicho subsistema se utilizan para su descripción, solo aquellas detectadas o consideradas relevantes.

Resulta evidente que para estudiar la regulación de la glucemia, por ejemplo, no resultaría práctico hacer una descripción de la localización de cada molécula de glucosa en la sangre a modo de distribución espacial. Para este estudio lo que resulta útil, y por lo tanto lo que nos permite proporcionar resultados, es el uso de la concentración de glucosa en sangre aunque sepamos que se trata de un dato promediado y por lo tanto inexacto. Pero esto conlleva asumir que la ley planteada será igualmente inexacta, aunque lo suficientemente aproximada para ser práctica y permitir la explicación y la predicción. A priori, este enfoque puede parecer indistinguible de la visión neo-mecanicista (Machamer, Darden y Craver 2000, Glennan 1996, Craver y Bechtel 2007), pero como veremos, hay diferencias sustanciales.

\section{Reinterpretando los pilares de la emergencia}

Basándonos en lo desarrollado anteriormente, es posible explicar los cuatro pilares de Klee sin acudir a la emergencia. Si consideramos el mundo como una sucesión de niveles anidados en función de la selección arbitraria de ciertos criterios de cuasi-descomponibilidad, y si para explicar un nivel consideramos en mayor o menor medida los niveles inferiores como cajas negras es perfectamente comprensible la existencia de la sensación de emergencia.

\section{a) Impredecibilidad}

La capacidad de predicción es explicable tanto desde la perspectiva emergentista como desde la reduccionista. Para hacerlo desde el primer enfoque, sus partidarios deberían demostrar que la impredecibilidad se basa en que las propiedades son debidas a la estructura y que ésta no depende de sus componentes, algo que aún no han hecho (Klee 1984, 51). Mill ya era consciente de que la impredecibilidad podría ser solo ignorancia, así como planteaba la posibilidad de deducir todo hasta el nivel químico, donde encontraba una brecha, y continuar con la predicción completa desde ese nivel (Mill 1843, 269). Eliminado este salto, la predicción podría ser completa. Spaulding también lo veía así cuando decía que «es una cuestión abierta si esta imposibilidad se debe a la estructura de la existencia, o a nuestra ignorancia $\gg$ (Spaulding 1912, 241).

La modelización que cada ciencia hace al trabajar con las simplificaciones de los intervalos ampliados de cuasi-descomponibilidad está detrás de la impredecibilidad, máxime cuando somos ignorantes del grado, e incluso de su existencia. También puede darse el caso de que lleguemos a predecir dicha propiedad de forma no identificable desde una perspectiva superior. Lo primero tiene como solución una mayor colaboración entre las ciencias 
implicadas en los IA, lo que traerá un conocimiento más detallado de los componentes y de sus relaciones. Un ejemplo es la dificultad de extrapolar los resultados obtenidos en la experimentación in vitro a sistemas in vivo, no solo por la influencia de la disolución, sino por los efectos de la multitud de moléculas que forman el medio celular. Esta aglomeración molecular (macromolecular crowding) desconocida hasta muy recientemente (Hervás y Navarro 2011,89), nos ha permitido saber que la célula, y por extensión el organismo completo, es un trabado de moléculas siendo el disolvente poco más que una capa situada entre éstas, de ahí que se esté empezando a trabajar en simulaciones que emulan las condiciones de alta concentración.

La segunda razón está relacionada con la necesidad de un análisis previo top-down que ya Morgan puso en evidencia (Morgan 1923, 5), pero no como consecuencia de la emergencia, sino para poder saber qué propiedades superiores son relevantes para nuestros intereses. Un ejemplo es el papel de la titina en la contracción muscular, más exactamente en la relajación de los sarcómeros de las fibras musculares. Desde un enfoque médico resulta interesante saber cómo se lleva a cabo dicha relajación, razón por la que se investiga a niveles moleculares descubriendo el papel de la titina, proteína que se desnaturaliza en la contracción y de cuya renaturalización depende la relajación muscular. El estudio top-down lleva a un análisis bottom-up mediante la observación de dominios aislados en proteínas individuales a través de técnicas de microscopía de fuerza atómica (AFM). Los análisis han hallado una correlación cualitativa directa entre la acción de la titina y la relajación cardiaca, relación que se hace patente cuando al modificar la proteína interfiriendo en su renaturalización se altera la relajación cardiaca (Alegre-Cebollada et al. 2012). La dificultad para establecer una relación cuantitativa es debida, entre otras cosas, a que los análisis en el estudio según las técnicas AFM no reproducen el entorno in vivo.

Una consecuencia de la impredecibilidad sería la dificultad de llevar a cabo una reducción teórica al estilo nageliano (Nagel 1961, 443-520). Como quiera que las leyes establecidas sobre las regularidades observadas en los niveles superiores están basadas en datos modelizados, estas serán a su vez leyes simplificadas y por consiguiente leyes-modelo. Por tanto, y de igual forma que los estados de diferente nivel no son comparables, las leyes de distinto nivel tampoco lo serán, lo que implica una imposibilidad en la práctica de realizar la reducción teórica. Sin embargo, esta afirmación no es una renuncia a un reduccionismo teórico, sino a su puesta en práctica habida cuenta de la inexactitud de las leyes superiores. No se puede establecer una identidad entre una ley inexacta y una exacta. Sin embargo, no hay ninguna razón, más allá de la afirmación de la emergencia, que impida deducir una ley de un nivel superior a partir de leyes de niveles inferiores siempre y cuando se dispusiera de la capacidad suficiente. No obstante, es cierto que salvo algún éxito parcial, esta forma de reducción no ha dado los resultados esperados. La idea de leyes-puente que conecten leyes inferiores con leyes superiores parte de una dificultad de planteamiento que tampoco puede resolverse con la corrección de Schaffner (Schaffner 1974, 617-618), como ya señalaron Kuhn o Feyerabend (Rosenberg 2007, 350).

\section{b) Novedad cualitativa}

El aspecto de la novedad cualitativa, a pesar de ser el más diferenciador de la emergencia, es quizá el más sencillo de explicar desde un enfoque reduccionista. Cada intervalo de cuasidescomponibilidad proporciona una perspectiva diferente, y las propiedades de cada uno de 
los niveles las describimos desde esa óptica. Por ejemplo, una molécula de $\mathrm{H}_{2} \mathrm{O}$ viene determinada por la configuración electrónica de sus hidrógenos y su oxígeno, que se encuentran en un nivel inferior. Pues bien, al nivel de la molécula se pueden observar ciertas propiedades novedosas que no pueden tildarse de cualitativamente novedosas pues son deducibles desde sus componentes. La «forma» de la molécula o su polaridad son propiedades del intervalo y son perceptibles solo a ese nivel. Además, hay que considerar que al tiempo que aparecen estas propiedades desaparecen las propias de los átomos individuales, sin que esto parezca ser un problema para el emergentismo. Si introducimos una segunda molécula de óxido de hidrógeno y las aproximamos lo suficiente como para que puedan interaccionar veremos que pueden bien formar un enlace covalente coordinado dando como resultado un ion hidronio $(\mathrm{H} 3 \mathrm{O})^{+}$y un ion hidróxido $(\mathrm{OH})^{-}$, bien atraerse formando una unión débil llamada enlace de hidrógeno $\left(\mathrm{H}_{2} \mathrm{O}\right)_{2}$ o bien no interaccionar. De nuevo hay novedades, tanto objetos (hidronio e hidróxido), como propiedades (atracción electrostática), pero ambas explicables desde las propiedades del intervalo inferior.

Demos un salto cuantitativo, y consideremos una cantidad superior a 10.000 de nuestras moléculas, número aproximado para que se manifiesten las propiedades del agua (Park 2013). Ahora no hay disyunción y pueden darse todas las situaciones anteriores al mismo tiempo. Además, una vez que una molécula adquiere uno de los estados, influye en el estado de las adyacentes dándose un proceso dinámico inducido. Nuestro nuevo $\varepsilon_{\mathrm{i}}$ nos sitúa en un nivel en el que el observador es del tamaño del total de las moléculas y su capacidad de observación le impide distinguir su individualidad y por tanto sus cualidades microscópicas. No obstante, observa sus consecuencias, como que esta sustancia se distribuye superficialmente ocupando todo el recipiente que lo contiene, a lo que denomina ser un líquido. Esto se corresponde con las moléculas formando enlaces de hidrógeno de manera que en vez de comportarse individualmente lo hacen como grupos y necesitan un nivel energético más elevado para escapar las unas de las otras y ocupar todo el volumen que las contiene, algo que nuestro observador llamaría ser un gas. Desde esta perspectiva se percibirá además que la superficie libre tiene cierta resistencia a ser penetrada, lo que denomina tensión superficial. Una tercera observación es la que se refiere a la cantidad de iones hidronio e hidróxido presentes, para lo que se introduce una magnitud a la que denomina $\mathrm{pH}$ y que no es sino una valoración promedio de la cantidad de iones hidronio presentes. Así, el ser líquido, la tensión superficial y el $\mathrm{pH}$ son propiedades que aparecen a partir de cierta cantidad de moléculas pero que son la consecuencia previsible de las características del nivel de organización previo. De aquí que no se pueda hablar de si una sola molécula de agua es líquida o gaseosa, del mismo modo en que no se podría hablar de si un determinado volumen de agua es o no líquido si el observador es del tamaño de una sola molécula, o al igual que en el ejemplo de Simon no se podía concretar la temperatura de un cubículo conociendo la de la casa, ni conocer la existencia de la casa desde el cubículo. Por esto, proponemos denominar a estas propiedades como propiedades de nivel, las cuales se manifiestan solo en un determinado intervalo sin acumularse. Así, si bien podría considerarse similar a la idea de emergencia de Mario Bunge quien afirma que una propiedad emerge cuando ninguno de sus componentes la posee (Bunge 2004, 32) se diferencia de esta postura en tanto en cuanto el filósofo argentino aboga por «la imposibilidad de comprender una totalidad a través del análisis de sus componentes y sus interacciones» $(2004,19)$. 


\section{c) Restricción o realizabilidad múltiple}

Klee (Klee 1984, 54) apuntaba el enfoque de Paul Weis (Weiss 1970), quien decía que si tenemos un sistema $S$ formado por $N$ partes y analizamos la variación-fluctuación de cada una de estas partes la variación del sistema es mucho menor de lo que se esperaría en función de las variaciones parciales. Esta idea es similar a la de las condiciones de contorno de Polanyi y las variantes ya citadas de Pattee y Rosen. Sin embargo, considerando el enfoque aquí planteado, esta restricción del nivel superior que se manifiesta en una aparente realizabilidad múltiple, resulta un mero artefacto de la simplificación hecha al considerar un intervalo de cuasi-descomponibilidad superior eliminando la distinción de los intervalos inferiores. Al observar solo ciertas características y de forma promediada, resulta evidente habrá menos descripciones macro que micro.

En el caso del agua líquida denominamos igual a muchos estados de ese conjunto de moléculas que sin ser exactamente los mismos, a nivel del observador se comportan como si lo fueran. Además, en función de la elección del $\varepsilon_{i}$, no haremos distinción ni siquiera de su composición. Si estamos describiendo el movimiento superficial de una masa de agua, nos dará igual de qué tipo de agua hablemos, pero si lo que consideramos es su cualidad de calmar la sed, sí haremos esta diferencia distinguiendo entre dulce y salada. En el caso más complejo de una molécula de hemoglobina, denominamos por igual a un amplio conjunto de moléculas composicional y estructuralmente diferentes que sin embargo cumplen solo cualitativamente la función de transportar oxígeno. La hemoglobina humana, solo en su cadena $\beta$ tiene 261 variedades, y si bien todas ellas cualitativamente intervienen en la captación y transporte del oxígeno, lo hacen de forma cuantitativamente diferente, bien aumentando, bien disminuyendo la afinidad por éste. A esto hay que añadir que muchas de ellas difieren en propiedades cualitativas. Por ejemplo, en la variedad Rothschild, el pequeño cambio del triptófano de la posición 38 de la cadena $\beta$ conlleva una rotación del tetrámero, ajuste que afecta a la cooperatividad, disminuyendo drásticamente su afinidad por el oxígeno y, como añadido, determina un sitio de unión para el anión cloruro en la zona de contacto entre las cadenas $\alpha 1$ y $\beta 2$ que implica una sensibilidad a la concentración de dicho anión que interfiere en la estabilidad de la molécula (Kavanaugh et al. 1992, 11).

Klee recurre (Klee 1984, 56) a la superveniencia de Jaegon Kim (Kim 1978) para rebatir la idea de la realizabilidad múltiple, sin embargo no cree que a los defensores de la restricción les preocupe esta explicación, pues tras su argumento subyace la idea de retro-control propio de la causación descendente. Tampoco Kim cree que la idea de superveniencia explique la emergencia de forma suficiente, pues la propia superveniencia es en sí misma inexplicable. «La conclusión es que la condición superveniencia en la emergencia simplemente equivale a la afirmación de que existe una covariación en principio inexplicable entre las propiedades supuestamente emergentes y sus propiedades de base» (Kim 2006, 555-556)

\section{d) Causación descendente}

Por último, e íntimamente ligado con la marca anterior, encontramos la causalidad descendente y, más generalmente la causalidad intranivel, considerada por Kim como la esperanza del emergentismo, que «no puede vivir sin la causación descendente, pero tampoco con ella. La causación descendente es la "raison d'être" de la emergencia, pero bien puede llegar a ser lo que al final la socave» (Kim 2006, 548). Amparada en este concepto se encuentra la idea de retrocontrol de los niveles superiores ya citada de Polanyi y su importante derivación de la 
autopoiesis, así como, al menos parcialmente, la explicación por mecanismos y la intuitiva idea científica de la búsqueda de la causación en los niveles inferiores.

Un aspecto evidente de la causalidad es que las causas anteceden a sus consecuencias, por lo que éstas deben encontrarse en la línea de tiempo pasado de su efecto. Así una situación temporalmente determinada de un nivel fundamental $F_{0}$ estará causada por otra previa $F_{-1}$. Si nuestra elección del criterio de cuasi-descomponibilidad nos hace estar analizando el fenómeno desde el nivel macroscópico, veremos que la situación $M_{0}$ está causada por una previa $M_{-1}$. Lo mismo nos valdría para cualquier nivel mesoscópico $m_{n}$. Como quiera que la situación $F_{0}$ y $M_{0}$ mantienen entre sí una relación de identidad, sus causas $F_{-1}$ y $M_{-1}$ también serán idénticas y por lo tanto no se puede hablar de causación internivel, ni ascendente ni descendente al no poder ser algo causa de sí mismo. Sin embargo, la idea de causalidad intranivel es muy fuerte (Craver y Bechtel 2007, 547) y esta intuición debe poder explicarse a fin de eliminar la subyacente circularidad de las causas.

Si observamos la causalidad de un acontecimiento A desde dos niveles distintos, y estamos interesados en la relación intranivel, es comprensible que analicemos aspectos diacrónicos como si fueran sincrónicos. Supongamos que queremos analizar la causa de $M_{0}$ en un nivel inferior, como quiera que esta situación es idéntica a $F_{0}$, su verdadera causa es la situación $F_{-1}$ que se corresponde con $M_{-1}$. Pero al no considerar el $d t$ transcurrido es fácil mezclar la explicación causal y la relación de identidad internivel y observar que $F_{-1}$ causa aparentemente $M_{0}$. Igualmente se puede tener la sensación de que $M_{0}$ mantenga una relación de condicionante sobre si misma visualizada como nivel microscópico, sin embargo, por la misma razón anterior, esto solo podría entenderse aparentemente sobre una situación micro posterior, y por lo tanto existir una causación descendente aparente entre $M_{0}$ y $F_{+1}$.

En la siguiente ilustración las flechas continuas simbolizan la causalidad intranivel, micro o macro, mientras que las flechas discontinuas refieren la aparente causalidad internivel, bien ascendente, bien descendente.

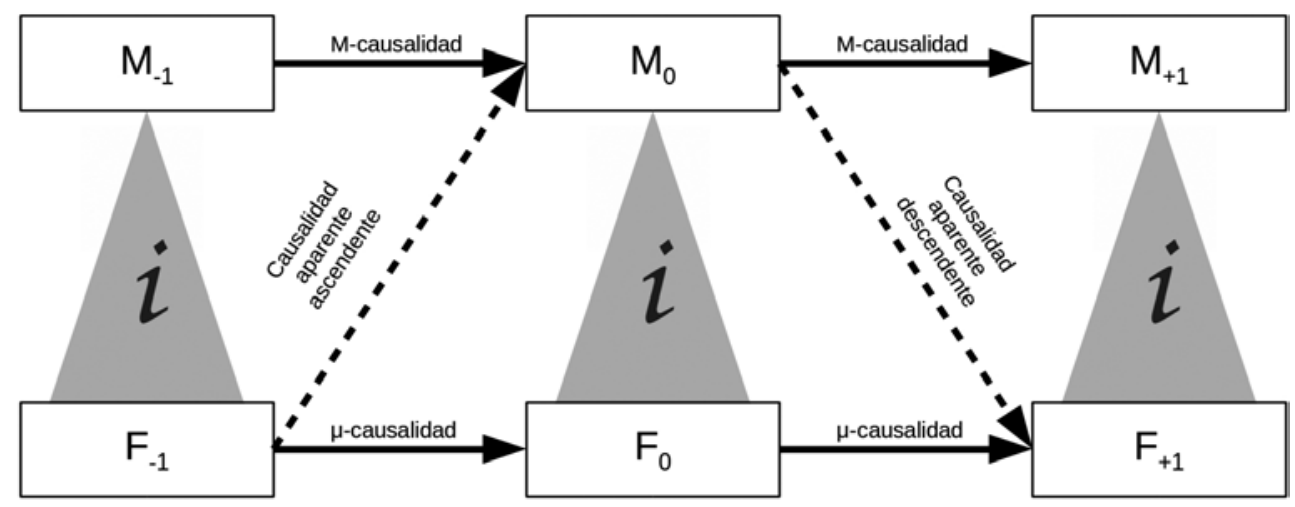

Ilustración 3. Macro-causalidad, micro-causalidad y causación internivel aparente. El cono gris representa la restricción y la letra $i$ la relación de identidad entre niveles

Así podemos explicar sencillamente la sensación de las causas subyacentes. Pensemos en la parte eléctrica de un coche híbrido. La intuición nos dice que este vehículo se mueve por la 
acción del motor eléctrico, lo que entendemos claramente como una causación ascendente. Según dónde situemos nuestro $\varepsilon_{i}$, podemos estar observando este coche desde un nivel en el que lo apreciamos completamente o desde otro en el que observamos sus componentes, como la batería o el alternador, sin visualizarlo por completo. En un momento inicial, tendremos un estado macro con su correspondiente estado micro. Si en un momento $t_{1}$, se produce un cambio en la batería, esto producirá una modificación en los demás componentes que observada desde el nivel macro consiste en el desplazamiento del coche. Si hacemos un análisis internivel, dará la sensación de que este cambio ha producido el movimiento del coche, pero si no cambiamos el nivel de observación, veremos bien a) la disposición de las piezas en $t_{0}$ da lugar a la disposición de las piezas en $t_{1}$, bien b) el coche en $t_{0}$ da lugar al coche desplazado en $t_{1}$.

De igual manera, intuimos que es el movimiento del coche lo que genera la electricidad que hace que el motor funcione. Aparentemente es una causación descendente, pero podemos explicarla de idéntica manera. Ahora nos fijamos en cómo cambia el alternador entre $t_{1}$ y $t_{2}$, algo que observado desde el nivel del coche nos dará los estados 1 y 2 . Si hacemos la misma observación cruzada, parecerá que el movimiento del coche (cambio $1 \rightarrow 2$ ) es el que modifica el alternador.

Cuando combinamos ambas observaciones, vemos que la batería inicia el movimiento, el movimiento carga el alternador y el alternador carga la batería, da la sensación de mecanismo cíclico, cuando en realidad es una espiral diacrónica a modo de volutas de avance temporal. Al no percibir claramente los diferenciales de tiempo queda la sensación de circularidad propia la idea de cierre causal.

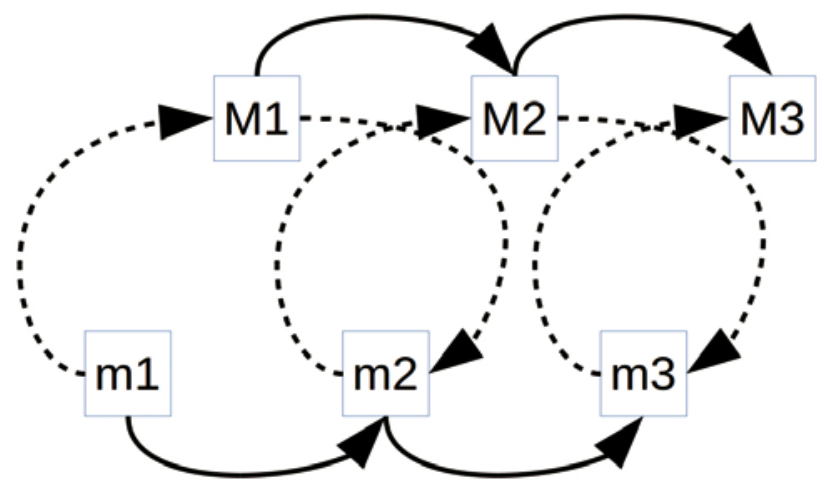

a

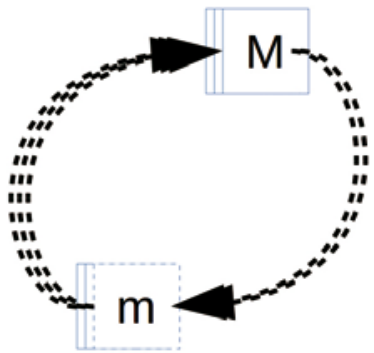

b

Ilustración 4. En a) se representa la causalidad cruzada entre niveles considerando el avance temporal tomando esta relación forma de voluta, mientras que en b) se elimina la referencia temporal tomando forma de ciclo con apariencia de causalidad descendente

El ejemplo anterior es muy sencillo, pero susceptible de complicarse. De igual manera que hemos descompuesto el coche en alternador y batería, podríamos descomponer cada uno de ellos en sus sub-componentes. La elección del criterio de cuasi-descomponibilidad podría ser, por ejemplo, $\varepsilon=\{$ componentes de una pieza $\}$, que iríamos agrupando modularmente 
o no (Simon 1962, 470). Más complicado, pero posible, es relacionar el comportamiento monógamo del ratón de campo con la densidad de receptores de vasopresina y oxitocina en las regiones subcorticales pálido ventral y núcleo accumbens (Churchland 2012, 62). En el caso anteriormente citado de la titina y la relajación muscular, los científicos se refieren a ella en forma de causalidad. Si una persona muere por un problema cardiaco relacionado con la relajación del miocardio, diremos que la causa de la muerte ha sido una malfunción de la titina, aunque se trata de una relación de causalidad ascendente aparente. El cambio de la titina entre $t_{0}$ y $t_{1}$ se observa como un cambio en el estado del sarcómero de $t_{0}$ a $t_{1}$ que, escalando niveles, veríamos como un cambio entre el individuo en $t_{0}$ a $t_{1}$ estando primero vivo y después muerto.

Este ejemplo sería similar al propuesto por Craver y Bechtel para dar una explicación mecanística de la causación internivel pero sin causa internivel (Craver y Bechtel 2007). Sin embargo ellos distinguen entre un ataque al corazón y una infección provocada por un virus, (2007, 456-457) al determinar que tanto el hospedador como huésped son individuos del mismo nivel aunque de diferente tamaño. Desde el punto de vista aquí desarrollado, no hay diferencia entre el virus, el sarcómero y demás componentes moleculares. El virus no interacciona con el organismo sino con ciertas moléculas, y una vez que lo hace, forma parte del mismo sistema y se debe considerar como un componente más. El enfoque de Craver y Bechtel no explica la integración del ADN vírico en los genomas de las células parasitadas cuando estos llevan a cabo un ciclo lisogénico que no se reactiva. También se podría considerar un medicamento como un individuo en el sentido que interacciona con la persona de la misma forma que el virus. Un león no interacciona con una población de cebras, sino con una cebra.

\section{Conclusiones}

A lo largo de este trabajo, se ha desarrollado un análisis de los cuatro pilares que sostienen la doctrina de la emergencia desde su origen a su resurgimiento en el último tercio del siglo Xx. Más de 100 años que se pueden resumir en las citadas impredecibilidad, novedad, restricción y causalidad descendente. Desde su primer desarrollo formal la emergencia ha estado vinculada a los niveles de organización y desde la perspectiva de establecer un criterio para determinar dichos niveles, es desde donde hemos pretendido explicarla. La ampliación de la cuasi-descomponibilidad de Simon y la idea de identidad entre niveles, más allá de la de composición que propone el mecanicismo, ayuda a establecer el nivel de estudio, y a definir la ciencia encargada de ellos en función del interés investigador. Por tanto afirmamos que la emergencia puede usarse exclusivamente con un enfoque heurístico.

Para un fin eminentemente práctico, bastaría con el establecimiento de leyes fenomenológicas, para lo cual estudiaríamos exclusivamente el nivel de nuestro interés, tratándolo como una caja negra, tomando únicamente las entradas y salidas de las variables escogidas y estableciendo leyes-modelo de ese nivel. Sin embargo, si además pretendemos explicar dichos fenómenos, resulta necesario indagar en los niveles subyacentes, lo que conlleva abrir esas cajas. La ventaja derivada del conocimiento de los niveles superiores facilita la elección de las variables para establecer un modelo, y por consiguiente establecer mejor las leyes-modelo de ese nivel. 
Para satisfacer la necesidad de explicación, es decir, para aclarar cómo funciona un sistema formado por niveles de subsistemas anidados, resulta útil y aclaratorio recurrir a un tipo de explicación que trate a sus elementos como componentes mecánicos. Un mecanosistema ${ }^{3}$ será, por tanto, el conjunto de cajas que una ciencia encuentra al abrir la caja de su nivel y las relaciones establecidas entre ellas. Estas cajas están delimitadas por el criterio de cuasi-descomponibilidad inmediatamente inferior demarcado por la ciencia en cuestión. Estos mecanismos tendrán a su vez sub-mecanosistemas, cajas dentro de las cajas, que podrán abrirse para analizar su interior, recursivamente hasta llegar al nivel F. Igualmente, el sistema desde el cual partíamos será a su vez una caja dentro de otras que forman la caja $\mathrm{M}$. El uso de mecanosistemas como recurso explicativo tiene varios aspectos a desarrollar, como la naturaleza de sus componentes, sus límites, el grado de simplificación, la asunción de pérdidas, su representación, etc.

Por último vale la pena remarcar la necesidad de establecer dos líneas de investigación diferentes. Por una parte, la necesidad de resultados prácticos inmediatos da pie a no descender demasiados niveles y a centrar la búsqueda de resultados en la evidencia. Por otra parte, el hecho de entender los mecanismos últimos de los fenómenos nos ayuda a afinar los resultados evitando efectos indeseados originados en el tratamiento fenomenológico del problema, algo demasiado frecuente, por ejemplo en la medicina. Por lo tanto, a pesar de esta practicidad, no hay que renunciar al descenso en busca de la explicación en el nivel F.

\section{REFERENCIAS}

Alegre-Cebollada, Jorge, Pallav Kosuri, Jason Feng, Anna Kaplan, Alvaro Inglés-Prieto, Carmen L. Badilla, Jose M. Stockwell, Arne Holmgren, Fernández, y Brent R. Sánchez-Ruiz. 2012. Protein Folding Drives Disulfide Formation. Cell, n. ${ }^{\circ}$ 151: 794-806.

Alexander, Samuel. 1920. Space, Time, and Deity. Vol 2. London: Mcmillan.

Baylis, Charles A. 1929. The Philosophic Functions of Emergence. The Philosophical Review 38 (4): 372-84.

Blitz, David. 1992. Emergent Evolution: Qualitative Novelty and the Levels of Reality. Springer-Science + Business Media, B.V.

Broad, Charlie D. 1925. The Mind and Its Place in Nature. London: Routledge.

Bunge, Mario. 1973. La metafísica, epistemología y metodología de los niveles. En Las estructuras jerárquicas, editado por Lancelot Law Whyte, Albert G. Wilson, y Donna Wilson. Madrid: Alianza Universidad.

-. 2004. Emergencia y convergencia. Barcelona: Gedisa.

Campbell, Donald T. 1974. Downward Causation in Hierarchically Organized Biological Systems. In F. Ayala and T. Dobzhansky (eds.), Studies in the Philosophy of Biology, Berkeley, University of California Press, 179-86.

Campbell, Richard J. y Mark H. Bickhard. 2011. Physicalism, emergence and downward causation. Axiomathes, n. ${ }^{\circ} 21: 33-56$.

Churchland, Patricia S. 2012. El cerebro moral. Lo que la neurociencia nos cuenta sobre la moralidad. Barcelona: Paidós.

Craver, Carl F. y William Bechtel. 2007. Top-Down causation without top-down causes. Biology and Philosophy 22: 547-63.

Dirac, Paul A. 1929. Quantum mechanics of many-electron systems. Proceedings of the Royal Society of London $A$ 123: 714-33.

3 Lo denominamos mecanosistema para distinguirlo del mecanicismo clásico. 
Emmeche, Clauss, Simo Köppe y Frederik Stjernfelt. 1997. Explaining Emergence: Towards an Ontology of Levels. Journal for General Philosophy of Science 28: 83-119.

Etxeberría, Arantza y Jon Umerez. 2006. Organismo y organización en la Biología Teórica: ¿Vuelta al organicismo? Ludus vitalis XIV (14): 3-38.

Garrison, Fielding H. 1922. Historia de la medicina. Tomo II. Madrid: Calpe.

Glennan, Stuart S. 1996. Mechanisms and the Nature of Causation. Erkenntnis 44: 49-71.

Hervás, Manuel y José A Navarro. 2011. Transferencia electrónica al fotosistema I: in vivo versus in vitro. En Actas del XXXIV Congreso SEBBM, p, 89. Barcelona.

Kauffman, Stuart A. 1993. Origins of Order: Self-Organization and Selection in Evolution. Oxford University Press.

Kavanaugh, J.S., P.H. Rogers, D.A. Case y A. Arnone. 1992. High-resolution X-ray study of deoxyhemoglobin Rothschild 37 beta Trp-Arg: a mutation that creates an intersubunit chloride-binding site. Biochemistry 31(16): 4111-21.

Kekes, John. 1966. Physicalism. The identity theory and the doctrine of emergence. Phiylosophy of Science 33: 360-75.

Kim, Jaegwon. 1978. Supervenience and nomological incommensurables. American Philosophical Quarterly 15: $149-56$.

-. 2006. Emergence: Core ideas and issues. Synthese 151: 547-59.

Klee, Robert. 1984. Micro-Determinism and Concepts of Emergence. Philosophy of Science 51: 44-63.

Lewes, George Henry. 1874. Problems of Life and Mind, first series, 2 vols. London: Truebner \& Co.

-. 1877. Problems of Life and Mind, second series. Boston: J. R. Osgood.

López Corredoira, Martín. 2004. Contra el libre albedrío en el marco de las Ciencias Naturales contemporáneas. Thémata, revista de Filosofía, n. ${ }^{\circ}$ 32: 227-305.

Machamer, Peter, Lindley Darden y Carl F. Craver. 2000. Thinking about Mechanisms. Philosophy of Science $67(1): 1-25$.

Maturana, Humberto, y Francisco Varela. 1973. De máquinas y seres vivos. Autopoiesis: la organización de lo vivo. Editado por Editorial Lumen. $2^{a}$ Edicion. 1993. Buenos Aires.

Mayr, Ernst. 2005. Asi es la biología. Barcelona: Debate.

-. 2006. Por qué es única la biología. Buenos Aires: Katz.

Meehl, Paul E. y Wilfrid Sellars. 1956. The concept of emergence. En Minnesota studies in the philosophy of science, editado por Herbert Feigl y Michael Scriven, pp. 239-52. University of Minnesota Press.

Mill, John Stuart. 1843. A system of logic: ratiocinative and inductive. [8th, 1872] ed. London: Longmans, Green, Reader, and Dyer.

Mitchell, Sandra D. 2012. Emergence: logical, functional and dynamical. Synthese 185: 171-86.

Morgan, C. Lloyd. 1923. Emergent evolution. Editado por Williams y Norgate. $2^{\circ}$ edition (1927). London.

Nagel, Ernest. 1961. La estructura de la ciencia. Editado por Paidós. 1. ${ }^{a}$ ed. 2006. Barcelona: Surcos.

O’Connor, Timothy. 1994. Emergent Properties. American Philosophical Quarterly, n. ${ }^{\circ}$ 31: 91-104.

Park, Jeong-Hyuck. 2013. How many is different? Answer from ideal Bose gas. Journal of Physics. arXiv: 1310.5580.

Pattee, Howard H. 1971. Physical theories of biological co-ordination. Quarterly Reviews of biophysics 4 (23): 255-76.

-. 1982. Cell Psychology: An Evolutionary Approach to the Symbol-Matter Problem. Cognition and brain theory 5(4): 325-34.

Penrose, Roger. 1991. La nueva mente del emperador. Barcelona: Grijalbo.

Pepper, Stephen C. 1926. Emergence. Journal of Philosophy, n. ${ }^{\circ} 23: 241-45$.

Piiroinen, Tero. 2014. Three Senses of «Emergence»: On the Term's History, Functions, and Usefulness in Social Theory. Prolegomena: časopis za filozofiju 13(1): 141-61.

Polanyi, Michael. 1968. Life's Irreducible Structure. Science, New Series, 160 (3834): 1308-12.

Rosenberg, Alex. 2007. Reductionism in Biology. En Philosophy of Biology, editado por Dov M. Gabbay, Paul Thagard, y John Woods, pp 349-68. Amsterdam: Elsevier. 
Rosen, Robert. 1985. Anticipatory Systems. Oxford: Pergamon Press.

Schaffner, Kenneth. 1974. Reductionism in Biology. Proceedings of the Biennial Meeting of the Philosophy of Science Association 1974: 613-32.

Silberstein, Michael, y John McGeever. 1999. The Search for Ontological Emergence. The Philosophical Quarterly 49 (195): 182-200.

Simon, Herbert A. 1962. The Architecture of Complexity. Proceedings of the American Philosophical Society 106 (6): 467-82.

Spaulding, Edward G. 1912. The New Realism: Cooperative Studies in Philosophy. New York: The Macmillan Company.

-. 1918. The New Rationalism: The Development of a Constructive Realism upon the Basis of Modern Logic and Science Through the Criticism of Opposed Philosophical Systems. New York: H. Holt and Company.

Weiss, Paul. 1970. The living system: determinism stratified». En Beyond Reductionism, editado por Smythies y Koestler, 3-55. New York: The Macmillan Company.

Wimsatt, William C. 2000. Emergence as Non-Aggregativity and the Biases of Reductionism. Foundations of Science 5 (3): 269-97.

Emilio Cáceres Vázquez. Es licenciado en Ciencias Biológicas por la Universidad Complutense de Madrid, en Ciencias Ambientales por la UNED y máster en Filosofía Teórica y Práctica por la UNED. Actualmente está elaborando su tesis doctoral sobre Propiedades Emergentes. Es autor de varios libros de texto y de ensayo y profesor de enseñanza secundaria y de formación profesional.

Dirección: Departamento de Lógica, Historia y Filosofía de la Ciencia, UNED, Senda del Rey 7, 28040 Madrid, Spain. Email: emiliocaceres@epifumi.com

Cristian Saborido. Profesor del departamento de Lógica, Historia y Filosofía de la Ciencia de la UNED. Doctor en Filosofía por la Universidad del País Vasco / Euskal Herriko Unibertsitatea. Especializado en Filosofía de la Ciencia, Filosofía de la Biología, Filosofía de la Medicina y Bioética. Autor de varias publicaciones en estas áreas en diversas compilaciones y revistas internacionales de investigación.

Dirección: Departamento de Lógica, Historia y Filosofía de la Ciencia, UNED, Senda del Rey 7, 28040 Madrid, Spain. Email: cristian.saborido@fsof.uned.es 